\title{
Aarnoo Avaliação da atenção ao diabetes mellitus em Unidades de Saúde da Família de Petrolina, Pernambuco, 2011*
}

doi: $10.5123 /$ S1679-49742014000300015

\author{
Quality evaluation of diabetes mellitus care in Family Health Centres, Petrolina, \\ Pernambuco, Brazil, 2011
}

Venâncio de Sant'Ana Tavares

Colegiado de Enfermagem, Universidade Federal do Vale do São Francisco. Petrolina-PE, Brasil

Suely Arruda Vidal

Mestrado Profissional em Avaliação em Saúde, Instituto de Medicina Integral Professor Fernando Figueira, Recife-PE, Brasil

Fernando Antônio Ribeiro de Gusmão-filho

Mestrado Profissional em Avaliação em Saúde, Instituto de Medicina Integral Professor Fernando Figueira e Secretaria Executiva de Gestão do Trabalho e Educação na Saúde, Secretaria Municipal de Saúde, Recife-PE, Brasil

José Natal Figueroa

Diretoria de Pesquisa, Instituto de Medicina Integral Professor Fernando Figueira, Recife-PE, Brasil

Silvanise Rosendo de Lima

Serviço de Atenção Domiciliar, Secretaria Municipal de Saúde, Petrolina-PE, Brasil

\section{Resumo}

Objetivo: avaliar a qualidade da atenção aos portadores de diabetes mellitus nas Unidades de Saúde da Família de Petrolina, Pernambuco, em 2011. Métodos: avaliação normativa, tendo como padrão as normas do Ministério da Saúde. Para avaliação de estrutura e processo, realizou-se observação de 40 unidades de saúde e entrevistas com profissionais; para avaliação de resultado, utilizaram-se dados dos Sistemas de Informação sobre Mortalidade e Hospitalização. Resultados: em todas as 40 unidades havia consultórios individuais e fitas reagentes, em 32 havia glicosímetros e em 9, profissionais de nível superior treinados. Foi evidenciada falta de endocrinologistas na atenção secundária. Todas as equipes realizavam atividades educativas e 22 realizavam o exame dos pés. Entre os triênios pré (2004-2006) e pós-implantação (2007-2009) do programa de controle da diabetes não houve diferença significativa na ocorrência de hospitalizações e óbitos por complicações da diabetes mellitus. Conclusão: a atenção ao diabético foi classificada como de qualidade regular.

Palavras-chave: Avaliação em Saúde; Diabetes Mellitus; Saúde do Adulto; Saúde da Família; Atenção Primária à Saúde.

\begin{abstract}
Objective: to evaluate diabetes mellitus patient care quality in Family Health services in Petrolina-PE, 2011. Methods: A normative evaluation following Brazilian Health Ministry standard guidelines. 40 health centres were observed and health professionals were interviewed in order to evaluate structure and process. Mortality and Hospital Admissions System data were used to evaluate outcomes. Results: all 40 health centres were equipped with individual consulting rooms and reagent strips. 32 health centres had blood glucose meters and 9 bealth centres had trained university-level professionals. There were no endocrinologists in secondary care. All teams undertook educational activities and 22 performed feet examinations. There was no significant difference in hospitalization and mortality due to diabetes mellitus complications in the three-year periods prior (2004-2006) and following (2007-2009) diabetes control program implantation. Conclusion: diabetes care was classified as being of regular quality.
\end{abstract}

Key words: Health Evaluation; Diabetes Mellitus; Adult Health; Family Health; Primary Health Care.

\footnotetext{
* Artigo derivado da dissertação do Mestrado Interinstitucional em Saúde Materno-Infantil do Instituto de Medicina Integral Prof. Fernando Figueira (IMIP) e Universidade do Vale do São Francisco (Univasf) intitulada Avaliação da atenção ao diabético em unidades de Saúde da Família do Município de Petrolina-PE, defendida em 2012 por Venâncio de Sant'Ana Tavares no Instituto de Medicina Integral Prof. Fernando Figueira (IMIP).
}

Endereço para correspondência:

Suely Arruda Vidal - Rua Francisco da Cunha, 1910/102-B. Boa Viagem, Recife/PE. Brasil CEP:51020-041

E-mail: suely@imip.org.br 


\section{Introdução}

0 diabetes mellitus (DM) é uma doença crônica não transmissível de alta prevalência, distribuída globalmente, estimando-se que havia cerca de 177 milhões de portadores no mundo em 2000 , sendo a projeção para 2030 de 350 milhões de doentes. ${ }^{1}$ Segundo dados do Vigitel $2010^{2}$ (Vigilância de fatores de risco e proteção para doenças crônicas não transmissíveis por inquérito telefônico) para diabetes, no Brasil e igualmente no Nordeste, 5,6\% dos adultos (18 anos ou mais de idade) referiram serem portadores desta doença, estimando-se ainda que haja uma proporção de $30 \%$ a $60 \%$ de casos não diagnosticados. No estado de Pernambuco, a razão de prevalência, segundo um estudo realizado em 2007 nas diferentes regiões brasileiras, foi acima de $17 \%$ em todas as faixas etárias. ${ }^{3}$

O DM é um problema de saúde pública com alta morbimortalidade e alto impacto social e econômico, em virtude das graves complicações. O Ministério da Saúde (MS) do Brasil lançou, em 2001, o Plano de Reorganização da Atenção à Hipertensão Arterial (HA) e ao diabetes mellitus (DM) ${ }^{4}$ formalizado na Portaria GM/MS n ${ }^{0} 235$ de 20 fevereiro de 2001. ${ }^{5}$ Tal Plano ${ }^{4}$ objetivou reduzir e controlar os fatores de risco, mediante a reorganização da rede de serviços em todos os níveis de complexidade, garantir o acesso aos medicamentos normatizados e incentivar a promoção de estilo saudável de vida, e preconizou que cerca de $80 \%$ dos casos de DM tipo 2 deveriam ser assistidos no primeiro nível de atenção.

\section{Segundo dados do Vigitel 2010, no Brasil e no Nordeste, $5,6 \%$ dos adultos ( $\geq 18$ anos de idade) referiram serem portadores de diabetes.}

Como suporte, foi implantado, em 2002, o Sistema de Informação da Gestão Clínica da Hipertensão Arterial Sistêmica (HAS) e DM da Atenção Básica (HiperDia) ${ }^{6}$ para cadastramento e monitoramento dos portadores de HA e DM, e algumas publicações técnicas para orientação aos profissionais da Estratégia de Saúde Família (ESF), entre as quais, o Caderno de Atenção Básica n ${ }^{\circ} 16$ (DM). ${ }^{7}$ Este Plano foi implantado em todo o país e teve sua primeira avaliação em
2003, conduzida pelo próprio MS em conjunto com a Organização Pan-Americana da Saúde (OPAS) ${ }^{8}$

Outras avaliações da qualidade da assistência ao DM na atenção básica se seguiram, como, em Recife, Pernambuco, em 2006, ${ }^{9}$ e em Francisco Morato ${ }^{10}$ e Ribeirão Preto, ambas em São Paulo, ${ }^{11}$ avaliando 0 controle da doença após a implantação do Plano.

Este trabalho teve por objetivo avaliar a qualidade da atenção aos portadores de DM nas Unidades de Saúde da Família (USF) de Petrolina, município de médio porte do sertão de Pernambuco, Brasil, em 2011, buscando contribuir para o aprimoramento da intervenção e para 0 alcance das metas, e também, para o fortalecimento da cultura avaliativa no âmbito dos serviços de saúde. ${ }^{12,13}$

\section{Métodos}

Trata-se de um estudo transversal descritivo do tipo avaliação normativa, ${ }^{12}$ tendo como padrão o Plano de Reorganização da Atenção à HA e ao DM implantado em Petrolina em 2008 e o Plano Municipal de Saúde, que reproduz as recomendações do plano nacional.

Entre as abordagens utilizadas para avaliação, dispõe-se do tipo normativa, ${ }^{12}$ utilizando-se o modelo sistêmico desenvolvido por Donabedian, ${ }^{13}$ o qual se apoia na tríade Estrutura, Processo e Resultado, e pressupõe uma relação de causalidade entre elas. Compara o existente no mundo real com critérios e normas fundamentados nas boas práticas clínicas e, segundo o autor, é um caminho direto para avaliar a qualidade da assistência. ${ }^{12}$

Petrolina é uma cidade polo do sertão de Pernambuco, separada do estado da Bahia pelo rio São Francisco. Em 2010, tinha 293.962 ${ }^{14}$ habitantes e Índice de Desenvolvimento Humano (IDH) 0,697. ${ }^{15}$ Tem como atividades econômicas a fruticultura, a criação de bovinos e caprinos, e a produção de vinhos. ${ }^{14}$ Sua rede municipal de saúde até novembro de 2009 era constituída de 47 USFs e dois hospitais de alta complexidade: 0 Hospital Dom Malan, referência para a saúde materno-infantil para a região, incluindo o norte da Bahia; e o Hospital de Urgências e Traumas, também de referência regional nestas áreas e nas clínicas médica e cirúrgica, conforme o Cadastro Nacional de Estabelecimentos de Saúde (CNES).

Este estudo foi realizado em 2011; a população do estudo foi composta por médicos ou enfermeiros das 
Equipes de Saúde da Família (ESF), estabelecendo-se como critério de inclusão: equipe completa no momento da coleta dos dados e médicos e enfermeiros atuando na área há mais de seis meses. Dessa forma, 40 equipes, entre urbanas e rurais - de cada uma das quais se entrevistou um destes profissionais, aquele que se prontificou a responder ao questionário -, preencheram estes critérios.

Como fonte de informação, utilizou-se um instrumento construído e validado em $2007,{ }^{9}$ adaptado aos objetivos do estudo, contendo questões referentes às dimensões de Estrutura e Processo, aplicado em entrevista aos profissionais, o qual foi complementado com observação direta nas unidades.

Como proxy da dimensão de Resultados, foram selecionados os óbitos e hospitalizações por complicações de DM dos residentes no município, extraídos do Sistema de Informações Hospitalares (SIH) e do Sistema de Informação sobre Mortalidade (SIM), nos períodos de 2004-2006 e 2007-2009, disponibilizados no sítio do Datasus, ${ }^{16}$ para verificar se houve mudança entre os triênios pré e pós-implantação do Plano. Nesta análise, aplicou-se o teste de Mantel-Haenszel, com nível de significância de $\mathrm{p}<0,05$, utilizando-se 0 Stata 12.1.

0 julgamento da atenção ao diabético ocorreu de acordo com o percentual calculado a partir do somatório da pontuação obtida nas entrevistas em relação à pontuação máxima possível para cada dimensão, com base no Plano de Reorganização da Atenção à HA e DM. ${ }^{8}$ Fez-se a classificação de cada dimensão separadamente e no conjunto, de acordo com o percentual alcançado: boa, se obtivesse um percentual variando de $81 \%$ a $100 \%$; regular, de $61 \%$ a $80 \%$; e baixa qualidade da assistência, se menor ou igual a $60 \%$.

Este projeto foi aprovado pelo Comitê de Ética da Universidade Federal do Vale do São Francisco (UNIVASF), sob o Certificado de Apresentação para Apreciação Ética (CAAE) $n^{\circ}$ 0049.0.441.441-11. Todos os participantes foram informados acerca dos objetivos e assinaram o Termo de Consentimento Livre e Esclarecido (TCLE), garantindo-lhes o sigilo de suas identidades.

\section{Resultados}

No tocante à dimensão Estrutura das unidades de atenção básica de Petrolina (Tabela 1), verificou-se que as salas de atendimento e de espera estavam dentro dos padrões estabelecidos. Glicosímetros e medicações (hipoglicemiantes orais e insulina) necessários para o tratamento da DM também foram encontrados na maioria das 40 USF estudadas - 32 e 31, respectivamente. Proporções mais baixas se referiram à presença de médicos e enfermeiros treinados para 0 desenvolvimento do programa ( $n=9)$ e à existência do manual de normas do MS $^{7}$ de orientação para o manejo dos casos de DM, encontrado em apenas 16 unidades.

A Tabela 2 exibe parte dos dados da avaliação da dimensão Processo. Todos os profissionais relataram utilizar as normas do MS para o diagnóstico da DM, e a consulta médica de acompanhamento na rotina foi relatada em 35 equipes. Contudo, o seguimento do calendário de consulta diferenciado para os casos de DM com lesão em órgão-alvo e para os não aderentes ao tratamento era realizada apenas em 24 . Todas (40) referiam cumprir a totalidade das recomendações indicadas à consulta clínica - controle de peso e pressão arterial, solicitação dos exames laboratoriais padronizados, consultas de enfermagem -, porém 0 exame clínico dos membros inferiores e a realização do teste de sensibilidade nos pés foram relatados por 22 e 12 equipes, respectivamente. A orientação para alimentação e exercícios físicos e visita domiciliar pelos agentes comunitários de saúde foi relatada por todas as equipes (40).

De acordo com a Tabela 3, ainda na avaliação da dimensão Processo, todas as equipes informaram fazer o primeiro atendimento de urgência e manter atualizados os envios dos dados do Sistema de Informação da Atenção Básica (SIAB) e HiperDia, porém 30 desenvolviam atividades de educação em saúde com grupos de diabéticos; 32 monitoravam o comparecimento às consultas; 33 equipes realizavam busca ativa aos faltosos; e 34 utilizavam os dados para o planejamento das suas ações.

Na dimensão de Resultados, avaliada pelas hospitalizações e óbitos, não se observou diferença estatisticamente significante entre os triênios pré e pós-implantação do programa ( $\mathrm{p}=0,059 \mathrm{ep}=0,307$, respectivamente), conforme apresenta a Tabela 4.

A Tabela 5 apresenta a classificação da qualidade da atenção prestada à DM pelas ESF, segundo a dimensão Estrutura, como de baixa qualidade $(57,7 \%)$ e bom processo de trabalho $(87,3 \%)$, e conjuntamente, como regular $(77,0 \%)$. 
Tabela 1 - Adequação dos recursos físicos, materiais e humanos (dimensão Estrutura) das Unidades de Saúde da Família para atenção ao portador de diabetes mellitus $(n=40)$, Petrolina-PE, 2011

\begin{tabular}{|c|c|c|}
\hline Variáveis & n & $\%$ \\
\hline \multicolumn{3}{|l|}{ Recursos físicos } \\
\hline Consultórios montados para 0 atendimento & 40 & 100,0 \\
\hline Área coberta (sala de espera) & 37 & 92,5 \\
\hline Sala para reunião ou atividades educativas & 16 & 40,0 \\
\hline \multicolumn{3}{|l|}{ Recursos materiais (equipamentos e insumos) } \\
\hline Balança, fita métrica, estetoscópio biauricular e esfigmomanômetro & 40 & 100,0 \\
\hline Glicosímetro com fitas reagentes suficientes & 32 & 80,0 \\
\hline Monofilamento de nylon & 4 & 10,0 \\
\hline Hipoglicemiantes orais e insulina suficiente & 31 & 77,5 \\
\hline Material educativo & 5 & 12,5 \\
\hline Caderno nº 16 de Atenção Básica & 16 & 40,0 \\
\hline Fichas do SIAB e do HiperDia ${ }^{b}$ & 38 & 95,0 \\
\hline Manutenção de rotina dos equipamentos & 17 & 42,5 \\
\hline \multicolumn{3}{|l|}{ Recursos humanos } \\
\hline Médico ou enfermeiro treinado na ESF ${ }^{c}$ & 11 & 27,5 \\
\hline Médico ou enfermeiro treinado para HA e DM & 9 & 22,5 \\
\hline Médico ou enfermeiro com pós-graduação & 21 & 52,5 \\
\hline$A C S^{d}$ e técnicos de enfermagem treinados na ESF & 29 & 72,5 \\
\hline Total (máximo esperado = 600) & 346 & 57,7 \\
\hline
\end{tabular}

a) SIAB - Sistema de Informação da Atenção Básica

b) HiperDia - Sistema de Gestão Clínica da Hipertensão Arterial e Diabetes Melito da Atenção Básica

c) ESF - Estratégia de Saúde da Família

d) ACS - Agentes Comunitários de Saúde

Na consulta ao Cadastro Nacional de Estabelecimentos de Saúde (CNES), foi evidenciada a falta de endocrinologistas na atenção secundária para encaminhamento das pessoas que apresentassem DM grave e refratária ao tratamento ou com lesões importantes em órgãos-alvo.

\section{Discussão}

A qualidade da assistência à DM foi classificada como regular no município estudado, principalmente pela estrutura, ausência de profissionais treinados, distribuição irregular de medicamentos e falta de referência no nível secundário da atenção. Por sua vez, o manejo clínico se classificou como bom, por seguir as recomendações do Ministerio da Saúde, segundo relato dos profissionais.

Os achados da avaliação normativa fornecem aos gestores uma visão da operacionalidade do programa, das facilidades e dos nós críticos que entravam a boa qualidade da atenção. Possibilita corrigir de forma 
Tabela 2 - Adequação das atividades de diagnóstico e de acompanhamento (dimensão Processo) relatadas pelas Equipes de Saúde da Família na atenção ao diabetes mellitus ( $n=40)$, Petrolina-PE, 2011

\begin{tabular}{|c|c|c|}
\hline Variáveis & $\mathbf{n}$ & $\%$ \\
\hline \multicolumn{3}{|l|}{ Atividades de diagnóstico } \\
\hline Rastreamento do DM na comunidade & 33 & 82,5 \\
\hline Utilização das normas para diagnóstico & 40 & 100,0 \\
\hline Realização de TOTGa nas gestantes & 40 & 100,0 \\
\hline \multicolumn{3}{|l|}{ Atividades de acompanhamento } \\
\hline Consulta médica $(\mathrm{CM})^{\mathrm{b}}$ & 35 & 87,5 \\
\hline CM semestral em usuários controlados e sem lesão em órgão & 33 & 82,5 \\
\hline CM mensal em usuários controlados com lesão em órgão & 24 & 60,0 \\
\hline CM mensal em usuários não aderentes ou de difícil controle metabólico & 24 & 60,0 \\
\hline Solicitação dos exames preconizados & 40 & 100,0 \\
\hline Consultas de enfermagem & 40 & 100,0 \\
\hline Exame clínico de rotina nos membros inferiores e pés & 22 & 55,0 \\
\hline Realização do teste de sensibilidade nos pés & 12 & 30,0 \\
\hline Aferição do peso e TA' em todas as consultas & 40 & 100,0 \\
\hline Monitoramento da glicemia em todas as consultas & 33 & 82,5 \\
\hline Orientação na consulta para alimentação e exercício & 40 & 100,0 \\
\hline Realização de visitas domiciliares pelos ACS ${ }^{d}$ & 40 & 100,0 \\
\hline Solicitação dos exames preconizados & 40 & 100,0 \\
\hline Visita aos acamados por médico/enfermeiro & 33 & 82,5 \\
\hline Pacientes diagnosticados sem acompanhamento & 23 & 57,5 \\
\hline Total (máximo esperado = 720) & 592 & 82,2 \\
\hline
\end{tabular}

a) TOTG - teste oral de tolerância a glicose

b) $\mathrm{CM}$ - consulta médica

c) TA - tensão arterial

d) ACS - Agentes Comunitários de Saúde

rápida os problemas identificados, tanto no nível da gestão, quanto na prática assistencial. ${ }^{12,13}$

Contudo, por ser tratar de doença crônica de longo curso, o DM exige que, além dos gestores e dos profissionais de saúde, os portadores assumam um compromisso de corresponsabilidade para o efetivo controle da doença e para manutenção da qualidade de vida. ${ }^{9}$
Cabe aos gestores fornecer a estrutura adequada para o desenvolvimento do processo de trabalho dos profissionais, respeitando os princípios das boas práticas clínicas e o projeto terapêutico individual ajustado às condições clínicas de cada usuário, ${ }^{14} \mathrm{e}$, aos pacientes, agir com disciplina no autocuidado, aderindo à dieta, aos exercícios e ao tratamento medicamentoso. ${ }^{23}$ 
Tabela 3 - Adequação das atividades (dimensão Processo) de educação em saúde, monitoramento, atendimento de urgência, registro e uso das informações na atenção ao diabetes mellitus nas Equipes de Saúde da Família ( $n=40)$, Petrolina-PE, 2011

\begin{tabular}{|c|c|c|}
\hline Variáveis & $\mathbf{n}$ & $\%$ \\
\hline \multicolumn{3}{|l|}{ Educação em saúde } \\
\hline Realização de educação em saúde nos grupos de diabéticos & 30 & 75,0 \\
\hline Realização de ações educativas periódicas na comunidade & 40 & 100,0 \\
\hline \multicolumn{3}{|l|}{ Monitoramento } \\
\hline Monitoramento de comparecimento às consultas & 32 & 80,0 \\
\hline Monitoramento do atendimento ao usuário na referência & 33 & 82,5 \\
\hline Realização de busca ativa aos faltosos & 33 & 82,5 \\
\hline \multicolumn{3}{|l|}{ Atendimento às urgências } \\
\hline $1^{\circ}$ atendimento de urgência nas complicações ou intercorrência na USF e acompanhamento & 40 & 100,0 \\
\hline \multicolumn{3}{|l|}{ Informação } \\
\hline Consolidação e envio dos dados do SIABa e HiperDia ${ }^{b}$ & 40 & 100,0 \\
\hline Cadastramento no HiperDia atualizado & 36 & 90,0 \\
\hline Uso dos dados do SIABa e HiperDiab no planejamento de ações & 34 & 85,0 \\
\hline Discussão dos casos acompanhados & 28 & 70,0 \\
\hline Total (máximo esperado $=400$ ) & 346 & 86,5 \\
\hline
\end{tabular}

a) SIAB - Sistema de informação da Atenção Básica

b) HiperDia - Sistema de Gestão Clínica da Hipertensão Arterial e Diabetes Melito da Atenção Básica

Tabela 4 - Hospitalizações e óbitos por complicações da diabetes mellitus (DM) em pacientes acima de 40 anos de idade no município de Petrolina-PE, no período 2004 a 2006 e 2007 a 2009, Petrolina-PE, 2011

\begin{tabular}{|c|c|c|c|c|c|c|c|}
\hline \multirow{3}{*}{ Período/Eventos } & \multicolumn{3}{|c|}{ 2004-2006 } & \multicolumn{4}{|c|}{ 2007-2009 } \\
\hline & \multirow{2}{*}{$\frac{\text { Total }}{\mathbf{n}}$} & \multicolumn{2}{|c|}{ Complicações por DM } & \multirow{2}{*}{$\frac{\text { Total }}{\mathbf{n}}$} & \multicolumn{3}{|c|}{ Complicações por DM } \\
\hline & & n & $\%$ & & N & $\%$ & $\mathbf{p}^{\mathrm{a}}$ \\
\hline Hospitalizações & 3.745 & 101 & 2,7 & 6.798 & 144 & 2,1 & 0,059 \\
\hline Óbitos & 2.411 & 59 & 2,5 & 2.715 & 78 & 2,9 & 0,307 \\
\hline
\end{tabular}

Fonte:DATASUS - Sistema de Informações Hospitalares (SIH) e Sistema de informação sobre Mortalidade (SIM). Disponível em:http://www2.datasus.gov.br/DATASUS/index.php. Acesso:junho/ 2011. a) Teste de Mantel-Haenszel 
Tabela 5 - Classificação da qualidade da atenção ao diabetes mellitus na Estratégia de Saúde da Família quanto às dimensões Estrutura e Processo ( $\mathrm{n}=40$ unidades de saúde), Petrolina-PE, 2011

\begin{tabular}{lcccc}
\hline Dimensões & Pontuação prevista & Pontos obtidos & \% alcançado & Classificação $^{\mathrm{a}}$ \\
\hline Estrutura (E) & 600 & 346 & 57,7 & Baixa \\
Processo (P) & 1.120 & 978 & 87,3 & Boa \\
Conjunto (E+P) & 1.720 & 1.324 & 77,0 & Regular \\
\hline
\end{tabular}

a) Boa $=81 \%$ a $100 \%$; Regular $=61 \%$ a $80 \%$; Baixa $=$ menor ou igual a $60 \%$.

Segundo um grupo italiano de gestão do controle da DM tipo 2 no adulto, o treinamento dos profissionais de saúde em equipe é o elemento-chave no bom manejo das doenças crônicas e pré-requisito para incorporação das atividades recomendadas pelas políticas nacionais de saúde, associado à integração entre os níveis primário e secundário de atenção. ${ }^{17}$

A ausência do endocrinologista na atenção secundária, em Petrolina, limitou o acesso e o controle dos casos mais complexos, restando aos pacientes 0 atendimento hospitalar. Dificuldade semelhante foi encontrada por outros autores ${ }^{18}$ em quatro capitais brasileiras em 2009, revelando a desorganização da rede hierarquizada no âmbito nacional. Talvez este seja um dos motivos para a não redução do número de hospitalizações ${ }^{19}$ e óbitos por esta causa e suas complicações antes e após a implantação do Programa.

A distribuição irregular dos hipoglicemiantes orais, também identificada em outros municípios, ${ }^{11,14}$ incluindo a capital do estado, Recife, pode contribuir para 0 abandono do tratamento e para a instalação de complicações cardiovasculares e cerebrovasculares. Ciente do prejuízo em médio e longo prazo ao doente, à previdência social e ao sistema de saúde, o MS criou em maio de 2012 o Programa Aqui tem Farmácia Popular, ${ }^{20}$ que garantiu, mediante convênio com algumas farmácias privadas em todo o Brasil, os medicamentos padronizados para controle do DM e da hipertensão arterial, que desde então passaram a ser dispensados gratuitamente, condicionado à apresentação da receita médica. Esta iniciativa nacional busca resolver o problema da falta do medicamentos no país.

Contudo, há que se valorizar o esforço da gestão pela aquisição dos glicosímetros e das fitas reagentes, disponíveis em todas as unidades básicas. Trata-se de um equipamento padronizado segundo normas, neces- sário e muito útil para o conhecimento imediato dos níveis glicêmicos dos pacientes, e que guia a instituição terapêutica apropriada em tempo real.

Entre as atividades importantes no cuidado ao diabético está o exame dos pés, visando prevenir lesões mais profundas, infecções e amputações, que se mostrou negligenciada em algumas ESF de Petrolina, assim como de Ribeirão Preto (São Paulo, Brasil) ${ }^{11}$ e do México. ${ }^{21}$ É procedimento simples, de baixo custo, útil para orientação ao autocuidado e, quando associado ao teste de sensibilidade, pode melhorar a eficiência da atenção ao portador de DM em $50 \%{ }^{21}$

As orientações ao autocuidado, à alimentação saudável e à prática de exercícios físicos são particularmente importantes para o controle da doença, mas com pouca adesão, conforme foi verificado em dois estudos de revisão sistemática publicados em $2011^{22}$ e 2012. ${ }^{23}$ As atividades de educação em saúde podem ser realizadas individualmente, como informado pelos entrevistados do presente estudo, ou em grupo, o que favorece a troca de saberes entre usuários e a equipe de saúde ${ }^{23}$ com valorização das experiências dos próprios participantes, permitindo a todos um processo integrador para um melhor controle terapêutico da doença. As duas formas de atividades educativas são efetivas, porém a educação em grupo apresentou melhores resultados no controle glicêmico após seis meses de trabalho. ${ }^{24}$

Há necessidade de se fortalecerem metodologias dirigidas às atividades de educação em saúde em grupo nos programas de educação permanente, uma vez que nem todas as ESFs possuem capacitação nessa área e nem os integrantes foram habilitados em seus cursos de graduação para o desenvolvimento de tais práticas. ${ }^{25}$

As hospitalizações e óbitos por complicações da DM, utilizados como indicadores de resultado, se mostraram praticamente inalterados, possivelmente 
pela ausência de alguns recursos estruturais e pela falta de algumas ações necessárias ao controle da doença, identificadas na presente avaliação.

Entre as limitações do presente estudo, destaca-se a coleta de dados com apenas um dos profissionais de nível superior, pois sabe-se que, sem outra técnica de observação das práticas referidas por estes que permitisse a triangulação destas respostas e as dos usuários, por exemplo, podem-se produzir vieses de informação; mas, ainda assim, foram detectadas falhas na adequação da estrutura e na qualidade da assistência. Outra limitação diz respeito aos indicadores selecionados para se avaliar a dimensão Resultado, pois é reconhecido que a mortalidade é um indicador de impacto e que as complicações podem demorar até décadas para se manifestar; porém, por se tratar de uma primeira aproximação ao objeto neste município, e por serem estes os indicadores disponíveis nos sistemas de informação selecionados, seria possível que se aproximassem dos resultados esperados, visto que, se a doença fosse controlada no período estudado, o número de hospitalizações poderia diminuir e, consequentemente, diminuiriam os óbitos.

Toda avaliação é dinâmica e visa identificar os problemas existentes, subsidiando os gestores no planejamento de ações que corrijam os eventuais desvios na condução dos programas, com o objetivo de se fornecer atenção de qualidade ao usuário dos serviços. É possível que uma nova avaliação, em momento mais recente, mostre resultados diferentes,

\section{Referências}

1. Organização Pan-Americana da Saúde. Saúde nas Américas 2007 [Internet].Washington: Organização Pan-Americana da Saúde; 2007 [citado 2010 jan 29]. Disponível em: http://www1.paho.org/HIA/ index.html

2. Ministério da Saúde (BR). Secretaria de Vigilância em Saúde. Secretaria de Gestão Estratégica e Participativa. Vigitel Brasil 2010: vigilância de fatores de risco e proteção para doenças crônicas não transmissíveis por inquérito telefônico[Internet]. Brasília: Ministério da Saúde; 2011 [citado 2013 out 1].Disponível em: http://bvsms.saude.gov.br/bvs/publicacoes/ vigitel_2010.pdf

3. Dias JCR, Campos JADB. Diabetes mellitus: razão de prevalências nas diferentes regiões geográficas no por uma série de motivos - lançamento de novas estratégias como o Plano de Enfrentamento das Doenças Crônicas Não Transmissíveis, o Programa Farmácia Popular, novos sistemas de informação (e-SUS Atenção Básica), avanços técnicos e troca de profissionais das equipes.

Como conclusão e contribuição desta avaliação, recomenda-se a incorporação do exame clínico dos pés - considerado atividade com boa relação custo-efeito - à rotina de assistência, para melhoria da qualidade da atenção ao DM em Petrolina, bem como ênfase nas atividades educativas em grupo, como incentivo ao autocuidado pelo diabético. Para isso são necessários treinamento e supervisão, assim como a reorganização da rede, a fim reduzir as hospitalizações e os óbitos.

\section{Contribuição dos autores}

Tavares VS, Vidal SA, Gusmão Filho FAR participaram da concepção e delineamento do estudo, análise e interpretação dos dados, redação e revisão crítica relevante do conteúdo intelectual do manuscrito.

Figueroa JN participou da análise e interpretação dos dados.

Lima SR participou da concepção e delineamento do estudo.

Todos os autores aprovaram a versão final do manuscrito e são responsáveis por todos os aspectos do trabalho, incluindo a garantia de sua precisão e integridade.

Brasil, 2002-2007. Cienc Saude Coletiva [Internet]. 2012 jan [citado 2013 set 27];17(1):239-44. Disponível em: http://dx.doi.org/10.1590/S141381232012000100026

4. Ministério da Saúde (BR). Secretaria de Políticas Públicas. Plano de reorganização da atenção à hipertensão arterial e ao diabetes mellitus. Rev Saude Publica [Internet]. 2001 [citado 2013 set 16];35(6):585-8. Disponível em: http://www.scielo. br/pdf/rsp/v35n6/7073.pdf

5. Ministério da Saúde (BR). Legislação federal da saúde: diabetes e hipertensão [Internet]. Brasília; 2006 [citado 2012 abril 9]. Disponível em: http:// bvsms.saude.gov.br/bvs/legislacao/diabetes_ legislacao.php 
6. Brasil. Ministério da Saúde. Portaria conjunta ${ }^{0}$ 002, de 5 de março de 2002 [Internet]. Brasília (DF); 2002 mar 5 [citado 2012 abril 9]. Disponível em: http:/hiperdia.datasus.gov.br/manuais/ portariaconjunta002.doc

7. Ministério da Saúde (BR). Secretaria de Atenção à Saúde. Departamento de Atenção Básica. Diabetes mellitus [Internet]. Brasília: Ministério da Saúde; 2006 [citado 2010 jan4]. (Cadernos de atenção básica; $n^{\circ} 16$; Série A. Normas e manuais técnicos). Disponível em: http://189.28.128.100/dab/docs/ publicacoes/cadernos_ab/abcad16.pdf

8. Ministério da Saúde (BR), Organização PanAmericana da Saúde. Avaliação do plano de reorganização da atenção à hipertensão arterial e ao diabetes mellitus no Brasil [Internet]. Brasília: Ministério da Saúde; 2004 [citado 2012 jan 9]. (Série C. Projetos, programas e relatórios). Disponível em http://bvsms.saude.gov.br/bvs/publicacoes/diab.pdf

9. Pereira PMH. Avaliação da atenção básica para 0 diabetes mellitus na estratégia saúde da família [dissertação na Internet]. Recife (PE): Fundação Oswaldo Cruz, Centro de Pesquisas Aggeu Magalhães; 2007 [citado 2012 jan 9]. Disponível em: http://www. arca.fiocruz.br/handle/icict/3923

10. Paiva DCP, Bersusa AAS, Escuder MML. Avaliação da assistência ao paciente com diabetes e/ou hipertensão pelo Programa Saúde da Família do Município de Francisco Morato, São Paulo, Brasil. Cad Saude Publica [Internet]. $2006 \mathrm{fev}$ [citado 2010 nov 29];22 (2):377-85. Disponível em: http://www.scielo. br/pdf/csp/v22n2/15.pdf

11. Silva ASB, Santos MA, Teixeira CRS, Damasceno MMC, Camilo J, Zanetti ML. Avaliação da atenção em diabetes mellitus em uma unidade básica distrital de saúde. Texto Contexto Enferm [Internet]. 2011 jul-set [citado 2012 set 27];(20)3:512-8. Disponível em: http://www.scielo.br/scielo.php?script=sci arttext\&pid=S0104-07072011000300012\&lng=pt\&nr $\mathrm{m}=\mathrm{iso}$

12. Brousselle A, Champagne F,Contandriopoulos AP, Hartz Z, organizadores. Avaliação: conceitos e métodos. Rio de Janeiro:Fiocruz; 2011. Capítulo, A apreciação normativa. p. 77-94.

13. Donabedian A. The quality of care: how can it be assesed? JAMA [Internet]. 1988 Sep [cited 2010 Jan 29];260(12):1743-8. Available from: post.queensu. ca/ hh11/assets/applets/The_Quality_of_Care How_Can_it_Be_Assessed_-_Donabedian.pdf
14. Prefeitura Municipal de Petrolina (PE) [Internet]. Petrolina;2010 [citado 2013 set 27].Disponível em:http://www.petrolina.pe.gov.br/2010/

15. Atlas do Desenvolvimento Humano no Brasil. Perfil municipal: Petrolina, PE [Internet]; 2013 [citado 2013 set 27]. Disponível em: http://atlasbrasil.org. br/2013/perfil/petrolina_pe

16. Ministério da Saúde (BR). Departamento de Informática do Sistema Único de Saúde - Datasus [Internet].Brasília; 2013 [citado 2013 dez 10]. Disponível em: http://www2.datasus.gov.br/DATASUS/ index.php?

17. Maggini M. IGEA -a chronic disease management project for people with diabetes. Ann Ist Super Sanita [Internet]. 20090ct-Dec [cited 2013 Oct1];45(4):349-52. Available from: http://www. iss.it/binary/publ/cont/ISSA09_0021_2571_2009 _S_45_04_349352.pdf

18. Giovanella L, Mendonça MH, Almeida PF, Escorel S, Senna MCM, Fausto MCR, et al. Saúde da família: limites e possibilidades para uma abordagem integral de atenção primária à saúde no Brasil. CiencSaude Coletiva [Internet]. 2009 mai-jun[citado 2012 set 28];14(3):783-94. Disponível em: http://www. scielo.br/scielo.php?script=sci_arttext\&pid=\$1413$81232009000300014 \& \operatorname{lng}=\mathrm{pt} \& \mathrm{nrm}=$ iso

19. Alfradique ME, Bonolo PF, Dourado I, Lima-Costa MF, Macinko J, Mendonça CS, et al. Internações por condições sensíveis à atenção primária: a construção da lista brasileira como ferramenta para medir o desempenho do sistema de saúde (Projeto ICSAP - Brasil). Cad Saude Publica [Internet]. 2009jun [citado 2012 ago 29];25(6):1337-49. Disponível em: http://www.scielo.br/scielo.php?script=sci arttext\&pid $=$ S0102-311X2009000600016\&lng=pt\&nr $\mathrm{m}=\mathrm{iso}$

20. Brasil. Ministério da Saúde. Portaria no971 GM/ MS, de 15 de maio de 2012. Dispõe sobre o programa farmácia popular do Brasil. Diário Oficial da República Federativa do Brasil [Internet], Brasília(DF), 2012 maio 17 [citado 2012 ago 27]. Seção 1:58. Disponível em:http://www.brasilsus.com. br/legislacoes/gm/113251-971.html

21. Salinas-Martínez AM, Amaya-Alemán MA, ArteagaGarcía JC, Núñez-Rocha GM, Garza-Elizondo ME. Eficiencia técnica de la atención al paciente con diabetes en el primer nivel. Salud Publica Mex [Internet]. 2009 ene-feb [citado 2013 nov 29];51(1):48-58. Disponible en: http:// 
www.scielo.org.mx/scielo.php?pid=S0036$36342009000100010 \&$ script=sci_arttext

22. Umpierre D, Ribeiro PB, Kramer CK, Leitão CB, Zucatti ATN, Azevedo MJ, et al. Physical activity advice only or structured exercise training and association with HbA1c levels in type 2 diabetes: a systematic review and meta-analysis. JAMA [Internet].2011 May [cited 2011 Nov 29];305(17):1790-9. Availablefrom: http://jama.jamanetwork.com/article. aspx?articleid $=899553$

23. Assis LC, Simões MOS, Cavalcanti AL. Políticas públicas para o monitoramento de hipertensos e diabéticos na atenção básica, Brasil. Rev Bras Pesqui Saude[Internet]. 2012 abr-jun [citado 2013 out 20];14(2):65-70. Disponível em: http://periodicos. ufes.br/RBPS/article/viewFile/4189/3313
24. Torres HC, Franco LJ, Stradioto MA, Hortale VA,Schall VT. Avaliação estratégica de educação em grupo e individual no programa educativo em diabetes. Rev Saude Publica [Internet]. 2009abr [citado 2013 out 20];43(2):291-8. Disponível em: http://www. iss.it/binary/publ/cont/ISSA09_0021_2571_2009 _S_45_04_349_352.pdf

25. Cavalcante MGS, Samico I, Frias PG, Vidal SA. Análise de implantação das áreas estratégicas da atenção básica nas equipes de Saúde da Família em município de uma Região Metropolitana do Nordeste Brasileiro. Rev Bras Saude Matern Infant [Internet]. 2006 outdez [citado 2013 out 25];6(4):437-45. Disponível em: www.scielo.br/pdf/rbsmi/v6n4/11.pdf.

Recebido em 28/11/2013

Aprovado em 25/05/2014 